\title{
Research on Image Nonlocal Denoising Algorithm based on Wavelet Decomposition
}

\author{
Jing Zhang \\ Dongchang College of Liao Cheng University, Liaocheng 252000, China \\ dcxydzx@126.com
}

\begin{abstract}
In the field of image denoising, nonlocal image denoising algorithm is a nonlinear, space average denoising algorithm, it will not cause boundary blurred, it is an effective denoising algorithm. But its application still has limitations because of it taking much longer time, in this paper, the method was improved, image signal can be divided into high frequency and low frequency part using wavelet decomposition, nonlocal denoising algorithm is used in low-frequency approximate signal, for high frequency detail signals using wavelet filtering method for denoising. The experimental results show that the method improves the speed of image processing and has good practical value.
\end{abstract}

Keywords: Image denoising; Nonlinear. Wavelet decomposition; Nonlocal algorithm

\section{Introduction}

Image processing, image analysis and processing, optimization, make it meet the requirements of visual and psychological, and other technology. At present most of the images are stored in digital, and image processing, image processing, in many cases, the index word. In addition, based on the theory of the optical processing method still occupies an important position in the field of image processing. Traditional onedimensional signal processing methods and concepts of many still can be directly applied in image processing, such as noise reduction, quantitative, etc. Belong to $2 \mathrm{~d}$ image signal, however, compared to one dimensional signal, it has its own special surface, the means of processing and point of view should also be different.

Noise processing is a very important role in image processing. Image is often random signal intensity (can also be called "noise") by pollution. In order to make the image more vivid, the need for image denoising. How to reasonably to eliminate a lot of noise, to keep the image of the original of all kinds of information, and to strengthen, and suitable for the human eye observation, has always been a image processing one of the main topics of the research neighborhood. With the development of computer technology, to improve the performance of the CPU, the popularity of large screen display, people is higher and higher demand for all kinds of video image, so for the image denoising technology demands more and more is also high. How can get better results in a shorter period of time, the resolution of the larger image has always been of concern to people.

Image denoising in other ways also have many applications, such as image recognition, in the presence of various interference information, accurate to find key information contains noise in the image, and lay a foundation for the subsequent processing of the image, such as applications in remote sensing images, aerial image, and image monitoring.

In the traditional image denoising neighborhood, the thinking of image denoising is divided into two main direction[1], using the image spatial domain information in image denoising, or derived from the Angle of the signal, the image as a two-dimensional signal, the signal processing in the frequency domain for image denoising. In spatial domain image denoising, mainly around the pixel to be processed image information is used to 
estimate calculation, including bilateral denoising method is relatively classic, in many cases have good performance, but any way could not adapt to all of the situation, it also has many problems. Since it is a kind of linear denoising method, there is a loss of details and inevitable defects such as fuzzy boundaries too.

After bilateral denoising algorithm, Buades and proposed a new better denoising algorithm, called the Non Local Means denoising algorithm [2-3], referred to as "while NLM algorithm, and can be referred to as the Local average denoising algorithm, this algorithm will bilateral denoising is calculated in the object from the level of pixel to pixel level, which USES pixels around the whole piece of image information to represent the point of information, so that we can effectively reduce the large amount of noise under the condition of the center pixel is not credible. And can be seen from the name of the algorithm, its calculation is local, theoretically, should be in full search more similar pieces of information, but in the actual calculation process, is similar to the one based on image block distribution is concentrated this assumption, of the similar area in the process of the search is still limited in a reasonable range.

While NLM denoising algorithm of similarity between the block and block comparison, and searching are very time consuming process. Better denoising effect have been obtained at the same time, it also at the expense of computing time as the price. Because the algorithm of denoising effect in current denoising algorithms is very outstanding, so now the research on the algorithm focuses on certain assumptions and simplify the algorithm itself, make its while maintaining the denoising effect, reduce the computational complexity. Based on the above reasons, we put forward the new idea, it is based on while NLM denoising algorithm and wavelet decomposition of thought, to deal with the situation of the large amount of noise is more effective, and compared with the original while NLM, under the condition of guarantee treatment effect, can greatly reduce the processing time.

\section{Related Work}

\subsection{NLM Algorithm}

Generally speaking, each kind of denoising algorithm has some threshold value or the parameters need to set. Considering the content of the image differ in thousands ways, how to set up a generic parameters, or make the parameter at the time of different images can automatically obtain the optimal solution, the denoising algorithm in the case of different images to be able to have a better performance, also is a key problem in the study of algorithm.

While NLM denoising algorithm with bilateral denoising algorithm, weighted average of each pixel in the weighted average of the process, taking into account the local structure similarity, thus achieved than bilateral denoising algorithm better denoising effect. While NLM denoising algorithm the basic idea is [4-7]: the current processing by the entire image, the pixel value of all the similar pixels and its structure by weighted average. For the weight of each pixel values, to the pixel as the center of the image subblock (usually take $7 \times 7$ window size) and between the current pixel as the center of the image sub-block, after using the Gauss weighted Euclidean distance to depict, weight eventually is equal to the negative exponential function value of the Euclidean distance.

Considering the local image sub-block factors, in the center of the estimated that the current pixel values, on the local structure and its similar pixels of the weight is bigger, and different distributions of the noise on the image sub-block is random, so get rid of the noise by the weighted average can better.

The specific calculation process as follows [8-9]: define the image with noise is $\mathrm{v}=\{\mathrm{v}(\mathrm{i}), \mathrm{i} \in \mathrm{I}\}$, the image after removing the noise is $\mathrm{NL}[\mathrm{v}]$, the calculation process of each pixel I is: 


$$
N L[\mathrm{v}](\mathrm{i})=\sum_{j \in I} w(\mathrm{i}, \mathrm{j}) \mathrm{v}(\mathrm{j})
$$

In which $w(\mathbf{i}, \mathbf{j})$ is depends on the pixel $\mathrm{i}$ and $\mathrm{j}$ two pixel point as the center pixel similarity between sub-block (Gauss weighted Euclidean distance) weights.

The calculation formula of $w(\mathrm{i}, \mathrm{j})$ is:

$$
w(\mathrm{i}, \mathrm{j})=\frac{1}{Z(\mathrm{i})} \exp \left\{-\frac{\left\|v\left(\mathrm{~N}_{i}\right)-\mathrm{v}\left(\mathrm{N}_{j}\right)\right\|_{2, a}^{2}}{h^{2}}\right\}
$$

In which $Z(\mathrm{i})$ is:

$$
Z(\mathrm{i})=\sum_{j} \exp \left\{-\frac{\left\|v\left(\mathrm{~N}_{i}\right)-\mathrm{v}\left(\mathrm{N}_{j}\right)\right\|_{2, a}^{2}}{h^{2}}\right\}
$$

In the above formulas, $\left\|v\left(\mathrm{~N}_{i}\right)-\mathrm{v}\left(\mathrm{N}_{j}\right)\right\|_{2, a}^{2}$ is between pixel $\mathrm{I}$ and $\mathrm{j}$, pixels within them centered pixel sub-block corresponding pixels Gauss (standard deviation for a) weighted distance between the square, $v\left(\mathrm{~N}_{i}\right)$ is i centered around the image sub-block collection of pixels, $h$ is the parameters of the denoising, in general, the linear proportional relationship, and the standard deviation of noise into or $\mathrm{h}=10 \mathrm{a}$.

Through theoretical analysis and experimental contrast can know ${ }^{[10]}$, while NLM denoising algorithm of denoising effect under the condition of subjective and objective, is superior to other common denoising algorithms, such as Gauss denoising algorithm, anisotropic denoising algorithm, the general minimum error denoising algorithm, bilateral denoising algorithm, etc.

However, this method has a larger obvious flaws is algorithm calculation is too complex, the efficiency is not satisfactory. Although in practice has been adopted to decide the simplification and assumption, for example, a search for similar piece range from full to a larger window (usually a $21 * 21$ ). But on the whole, this method is time consuming compared to other methods. This has caused me to think how to reduce the calculation time.

\subsection{Wavelet Threshold Denoising Algorithm}

In the traditional denoising method is by noise interference signal through a filter, filter out the noise frequency part. However, this method for pulse signal and white Gauss noise, the nonstationary process such as the traditional method has some limitations. For this kind of signal, in the case of low SNR, through filtering processing, not only the SNR no big improvement, and signal to the original location of the information will be blurred out.

Denoising method based on wavelet transform, wavelet transform of the variable scale features, to determine the signal with a "concentration" ability. If most of the energy of a signal information is concentrated in wavelet minority wavelet transform domain coefficient, so their value must be greater than in the wavelet transform domain energy spread of the wavelet coefficient of signal and noise. This time, you can use the wavelet threshold method.

Before the choice of wavelet coefficient, according to certain rules ${ }^{[11,12]}$, the wavelet coefficients can be classified into two kinds: one kind is important, the rules of wavelet coefficient, and the other is as important, or the interference of wavelet coefficients. Normally, we use the absolute value of wavelet coefficient as the basis of classification of wavelet coefficients. The wavelet coefficient absolute value tends to zero, the means of the wavelet coefficient contains very little information, and these coefficients are most likely by the interference of noise. 
Processing threshold is the key to the choice of the threshold, if the min value is too small to filter the noise remains, but the threshold value if it is too big, important signals or is characteristic of the image will be filtered out, and cause deviation. From the intuitive, the selection of threshold value, to get the wavelet coefficients of the noise, the greater the threshold should be, the greater the most threshold selection process, is a set of wavelet coefficients, according to this set of statistical characteristics of wavelet coefficients, calculate its threshold.

Using the threshold value of signal or image denoising process, first we need to denoising signal or image for 11 layers of wavelet decomposition, the scale of the decomposed coefficients and wavelet coefficients of a vector. So-called soft threshold and hard threshold method, is carried out on the coefficient vector threshold transform, a new wavelet coefficient vector, and again by the wavelet reconstruction with the new wavelet coefficient vector and can get after denoising signal.

\section{Improved NLM Model}

In the general case. Digital image noise noise causes are diverse, and characteristic are different kinds of noise. All have a common characteristic. But the noise in different frequency bands and different channels (color image) will have different performance. For example, a certain amount of Gauss noise is mainly concentrated in the high frequency part of image, this also is the wavelet describs the main starting point of value to receie method, did it only for the high frequency of treatment: for digital camera photo. At the time of without any post-processing, red, green and blue (RGB) three color channels, blue channel is one of the largest channel noise contamination rate [13].

In the case of Gauss noise, after multiresolution decomposition, part of different frequencies of noise is different. For noise points, therefore, frequency of treatment or points of color channel has certain benefits, often can reduce processing time, or to different treatment for different parts, reduce the difficulty of processing, etc. This article discusses the different frequency processing is the case, the main point is the use of the characteristics of multi-resolution for original while NLM algorithm

In view of the noise in different frequency bands form, multiresolution analysis method is an efficient way. This method can effectively reduce the noise of the high frequency part of image. It will image is decomposed into different frequency bands, this approach than in the case of no decomposition, can give us more information of image information and noise.

First of all, for an input image containing noise, on the two-dimensional wavelet decomposition, decomposition can get information of two parts, the low-frequency approximate information $\mathrm{L}$ and high-frequency details of the $\mathrm{H}$, among them, the high frequency detail information and contains three parts: the level of detail, vertical details and diagonal detail information.

After then, for the decomposition of two pieces of information, due to the low frequency part of the approximate information contained more image content, the mix of noise and image information, and no more obvious distinguish, therefore, need for a decomposition, decomposition process is similar to the above situation. In the experiment, we found that the need to break down the number of layers related to image quality and features in our experiments, normally we only decomposed to the second floor. Decomposed to the bottom of the time, for the low frequency part, due to containing noise is less, so we do not make any processing to its: for the high frequency part we can according to common practice, using the way of wavelet threshold filtering noise. Then the two pieces of information through the combined wavelet inverse transformation.

For low frequency information merging step, we use while NLM denoising algorithm to its, get L1 '; Other high frequency information, we will according to common practice, also use way of wavelet threshold filtering noise. The most and inverse transformation, 
then get the final output image. Wavelet threshold is the key to determine the size of the optimal threshold. This approach for the high frequency part of the noise is very effective. Including the use of the wavelet threshold denoising method for the Bayes shrink denoising method, the calculation method of the threshold is as follows [14]:

$$
T(\mathrm{a})=\frac{a_{n}^{2}}{a_{s}^{2}}
$$

The molecules on behalf of the estimates of noise variance, the denominator represents the image signal variance estimation. After the wavelet decomposition by this formula of high frequency information filtering, who said its absolute value is less than the threshold coefficient contains less information, noise disturbance was more likely, so we need the score to filter (zero).

According to the different treatment of high frequency noise, we can get after processing the information of different frequencies, and then in the opposite order of the original decomposition reconstruction processing, and the final results after denoising image is obtained.

The proposed algorithm combines the basic framework of wavelet decomposition reconstruction, the wavelet threshold denoising method, while NLM denoising algorithm and carries on the improvement and implementation. Under the framework of this new algorithm cannot gay state noise for different processing, and achieve better denoising effect.

Take the current denoising effect is the best, to use an image alone while NLM algorithm are compared. Use while NLM algorithm alone not to distinguish between the frequency of the noise or type, are easy to remove some types of noise, such as concentrated in the high frequency part of the noise, and unified application while NLM algorithm, such as a result of the algorithm is an overall increase in time.

The method advantage is that in the basic keep denoising effect under the premise of computing resources can be effectively concentrated on the removal of the noise of the low-frequency approximate part and high frequency part with general wavelet threshold denoising method, its computation time compared with while NLM denoising algorithm of time will be a lot less.

In this paper, the method in the treatment of low frequency noise approximation part, due to the resolution of the low frequency approximation part is only $1 / 4$ of prior to decomposition of the original, so denoising algorithm implementation while NLM, processed by the pixel also decreased significantly, the corresponding processing time is reduced a lot. And in some special cases, consider the corresponding decrease during the processing of the size of the image sub-block and the size of the area in search for similar, it can also reduce will handle the computing time.

\section{Experiment and Analysis}

In order to do the contrast on data, we to manually add a series of experiments the image noise. Add noise to zero mean Gauss white noise. To deal with the noise in noise image, we will be calculated after denoising image and PSNR of the image before adding noise, as image of the judging criteria, which are defined as follows:

$$
\begin{gathered}
M=\frac{1}{\mathrm{~m} \times \mathrm{n}} \sum_{i=1}^{m} \sum_{j=1}^{n}\left(\mathrm{f}(\mathrm{i}, \mathrm{j})-N(\mathrm{i}, \mathrm{j})-X^{\prime}(\mathrm{i}, \mathrm{j})\right)^{2} \\
P N S R=-10 \lg \frac{M}{255^{2}}
\end{gathered}
$$

Among them, $\mathrm{M}$ is variance, as the peak signal-to-noise ratio, PSNR is the most common and most widely used the objective measurement method of the evaluation of image quality, but many experimental results show that PSNR unable to score and the 
visual quality of the human eye to see completely consistent, likely PSNR high looks worse than PSN - R lower instead. This is because the human eye vision for the sensitivity of the error is not absolute, the perceptual result will be influenced by many factors, resulting in changes (for example: the human eye to empty asked low frequency contrast sensitivity difference is higher, the sensitivity of the human eye to brightness contrast difference is relatively high colority, perception of the human eye to a regional results will be affected by the adjacent area around). Therefore, PSNR can only as a measure of image quality standard, the stand or fall of image is correlated with human's subjective feeling.

Table 1. PSNR Comparing of Filtered Image

\begin{tabular}{|c|c|c|c|c|}
\hline & $\begin{array}{c}\text { Amount of } \\
\text { noise }\end{array}$ & $\begin{array}{c}\text { Bilateral } \\
\text { denoising }\end{array}$ & $\begin{array}{c}\text { Original NLM } \\
\text { denoising }\end{array}$ & New method \\
\hline \multirow{3}{*}{ Lena } & $\sigma=10$ & 29.35 & 31.29 & 31.11 \\
\cline { 2 - 5 } & $\sigma=30$ & 28.65 & 29.65 & 29.34 \\
\cline { 2 - 5 } & $\sigma=50$ & 26.54 & 28.68 & 28.42 \\
\hline \multirow{3}{*}{ Pepper } & $\sigma=10$ & 29.87 & 32.51 & 32.26 \\
\cline { 2 - 5 } & $\sigma=30$ & 28.64 & 29.81 & 29.26 \\
\cline { 2 - 5 } & $\sigma=50$ & 27.69 & 28.63 & 28.16 \\
\hline \multirow{3}{*}{ House } & $\sigma=10$ & 29.28 & 30.54 & 30.68 \\
\cline { 2 - 5 } & $\sigma=30$ & 28.49 & 29.57 & 29.68 \\
\cline { 2 - 5 } & $\sigma=50$ & 27.19 & 28.68 & 28.71 \\
\hline
\end{tabular}

Table 5.1 we can see, we put forward a new method is compared with the classical bilateral denoising method, treatment effect on had the very big difference. In while NLM denoising algorithm compared with the original, statistically speaking, in a slightly less than a hour: when a relatively large data flat or slightly ahead. And both time-consuming, by contrast, we proposed a new method in image resolution is $512 \times 512$ computing time needed to about $360 \mathrm{~s}$, whereas the original while NLM denoising method under the condition of equal to $1425 \mathrm{~s}$. For a little bit small images, such as House, this scenario, a resolution of $256 \times 256$, our approach to the $89 \mathrm{~s}$, the original while NLM denoising method need $369 \mathrm{~s}$. Over time the test is in the dominant frequency of Pentium4 3.00 $\mathrm{GHz}, 512 \mathrm{MB}$ of memory on the machine.

Here we can see some effect from below comparison chart to look closely at the several denoising methods.

Figure 1 is to Lena figure, in the case of $\mathrm{a}=40$, can see clearly from the figure denoising methods. We can see that the bilateral denoising method can't good to remove a lot of noise. While NLM denoising method in the case of large amount of noise, on the PSNR value increase to a certain extent, but will look from actual human eye observation, some spots or block would happen. And we though in PSNR and while NLM basically unchanged, but in terms of macroscopic observation, our method is more suitable for human visual standards.

Figure 2 is Peppers the picture when $a=30$ denoising methods. Similar to table 1 in the performance of the various methods. We can observe that the prospect of the surface of the two green peppers, our method is more gentle than while NLM, while NLM has massive spots

Figure 3 is House this scene, the scene is the resolution of the smaller, you can see from the images, our method's handling of such noise is much better than while NLM.

It is can be seen from the above three groups of contrast experiment, we proposed methods though the PSNR compare with the original data while NLM has a tiny difference, but from the actual human visual effect, basic can achieve even more than 
while NLM denoising effect of the algorithm. Even in some cases, while a data was less, but the human eye $\sim$ better visual effect.

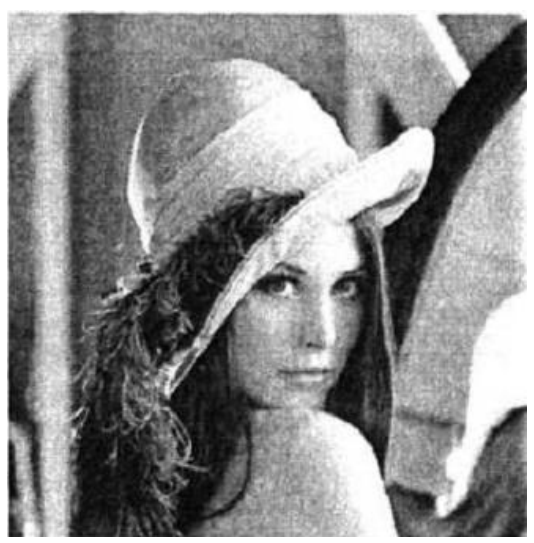

1. Original image

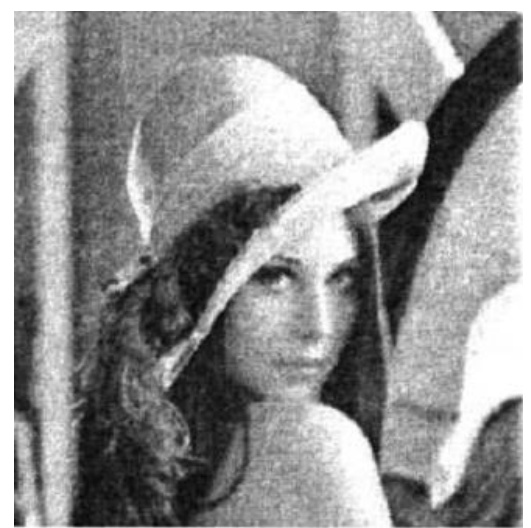

3. Bilateral denoising

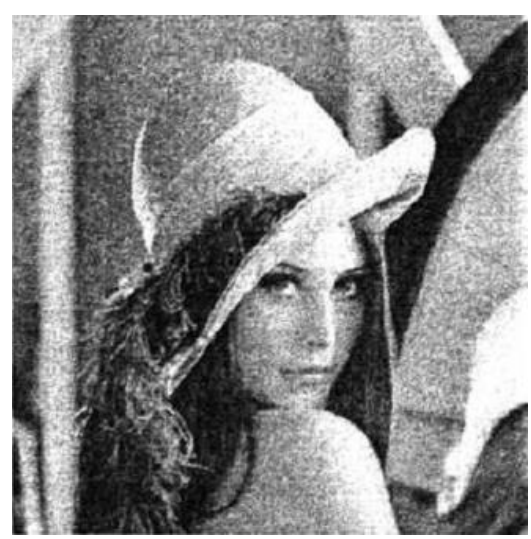

2. Image with noise

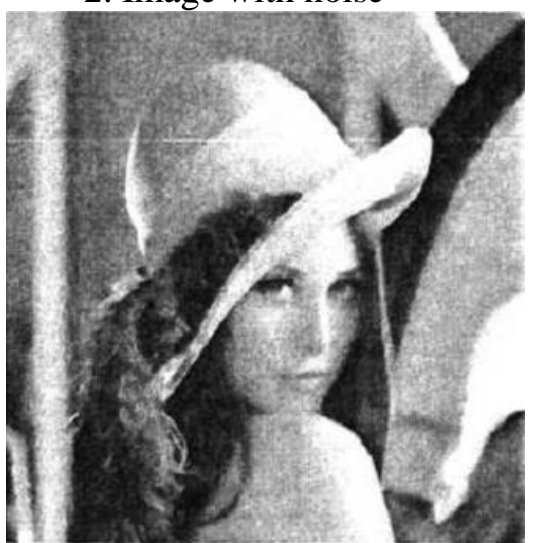

4. NLM denoising

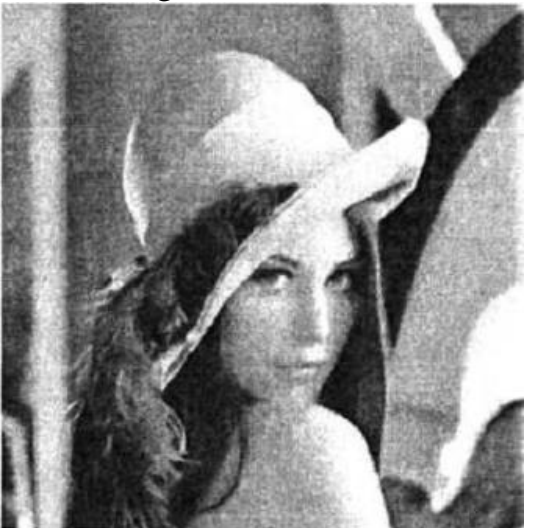

5. New method

Pic1. Lena image denoising comparing 

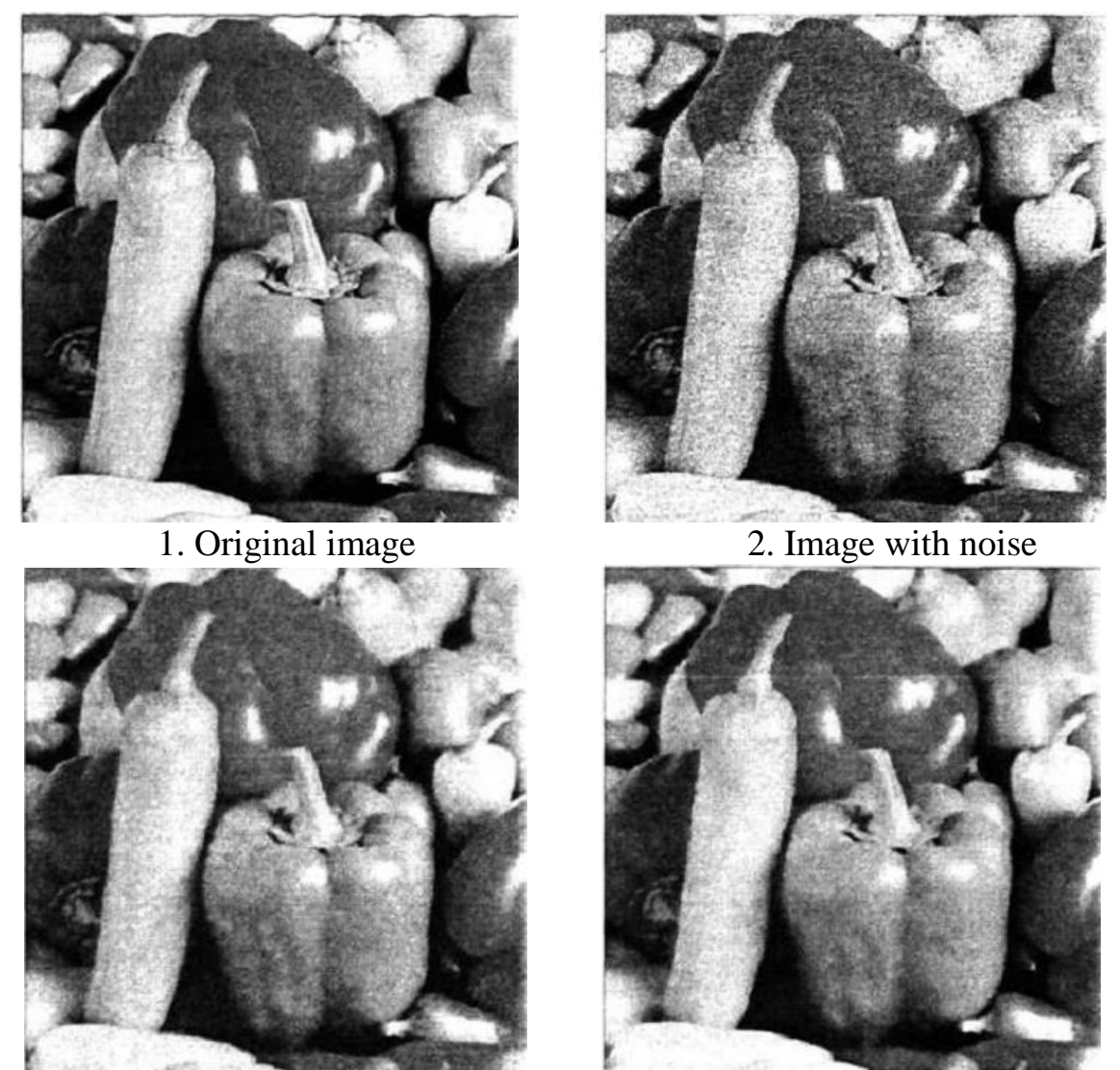

2. Image with noise

3. Bilateral denoising

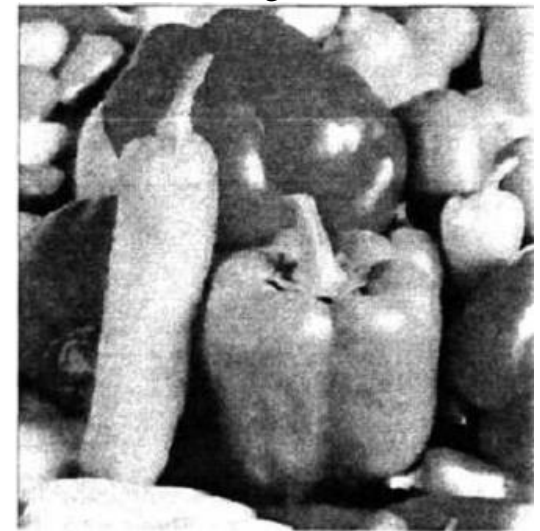

4.NLM denoising

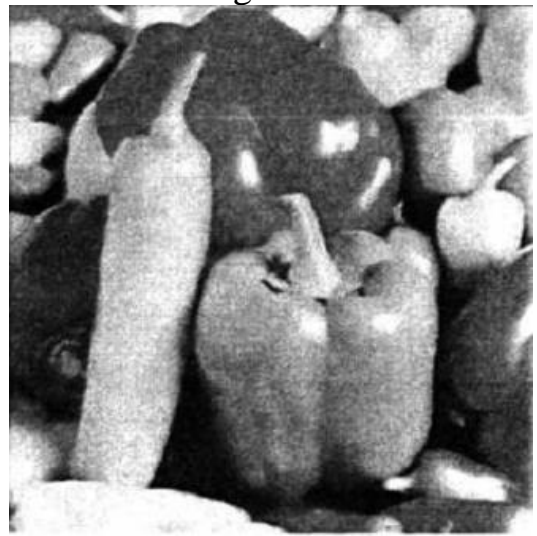

5. New method

Pic2. Pepper image denoising comparing

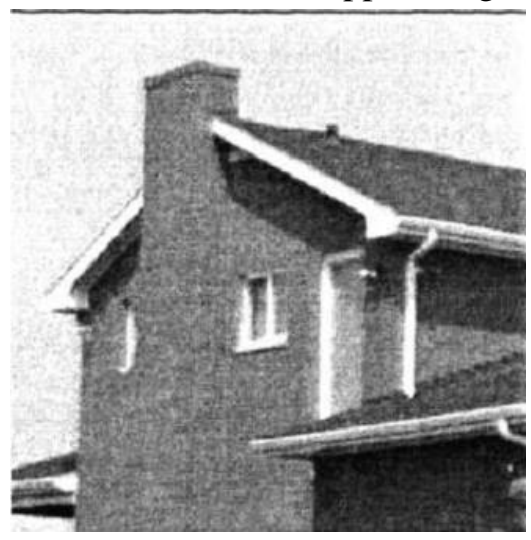

1. Original image

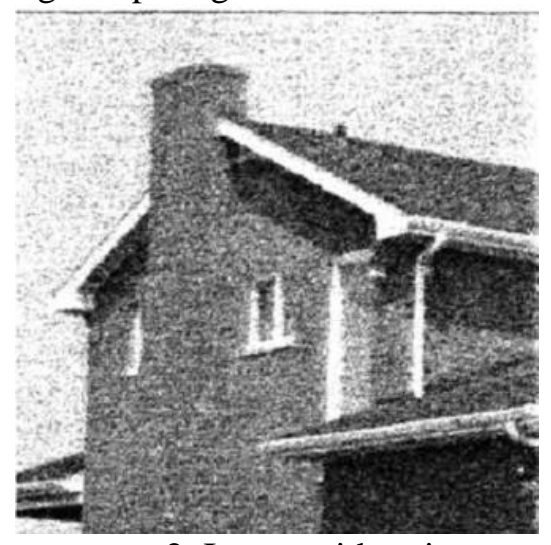

2. Image with noise 


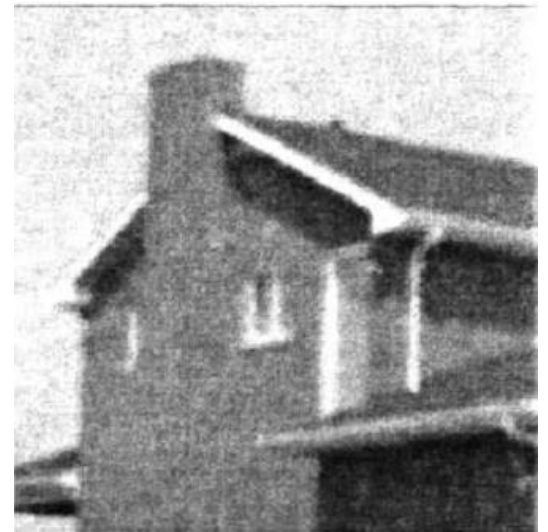

3. Bilateral denoising

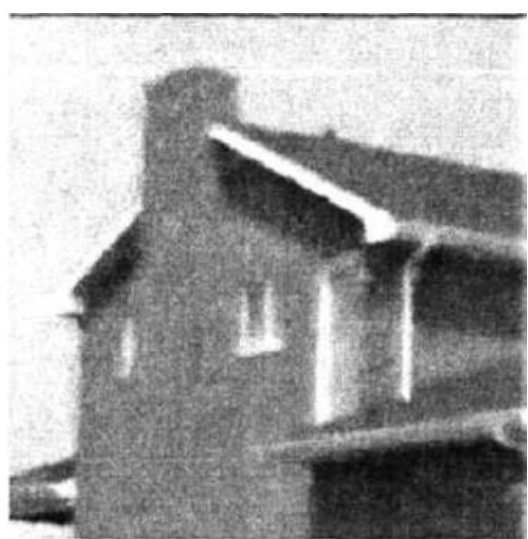

4.NLM denoising

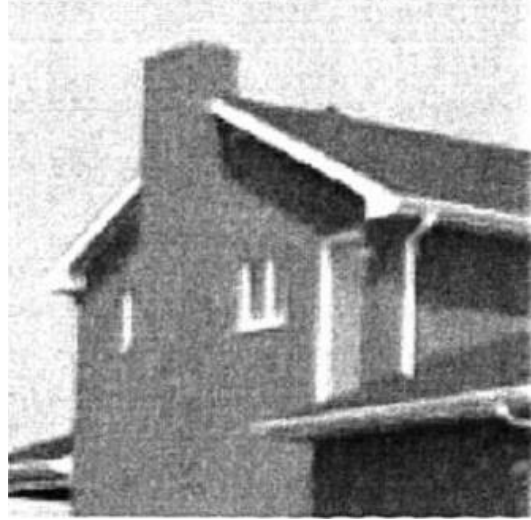

5. New method

\section{Conclusion}

Pic3. House image denoising comparing

In this paper, while NLM denoising method based on multi-resolution not only absorbed the advantages of the original while NLM processing, and the beneficial tool by using multiresolution analysis, the classification of the noise effectively can be met. For different noise and reasonable use of computing resources, easy to remove the noise using the wavelet threshold method, for use while NLM method is difficult to remove the noise, so that they can both time and effect of these two factors, the balance of its reach a compromise.

\section{References}

[1] O. Rockinger, "Image sequence fusion using a shift-invariant wavelet transform [C]", Image Processing, 2005, Proceedings, International Conference on. IEEE, vol. 3, (2005), pp. 288-291.

[2] S G. Chang, B. Yu and M. Vetterli, "Adaptive wavelet thresholding for image denoising and compression [J]”, Image Processing, IEEE Transactions on, vol. 9, no. 9, (2000), pp. 1532-1546.

[3] A. Buades, B. Coll and J M. Morel, "A review of image denoising algorithms, with a new one [J]", Multiscale Modeling \& Simulation, vol. 4, no. 2, (2005), pp. 490-530.

[4] J L. Starck, E J. Candès and D L. Donoho, "The curvelet transform for image denoising [J]", Image Processing, IEEE Transactions on, vol. 11, no. 6, (2002), pp. 670-684.

[5] J. Portilla, V. Strela and M J. Wainwright, "Image denoising using scale mixtures of Gausss in the wavelet domain [J]", Image Processing, IEEE Transactions on, vol. 12, no. 11, (2003), pp. 1338-1351.

[6] M. Elad and M. Aharon, "Image denoising via sparse and redundant representations over learned dictionaries [J]", Image Processing, IEEE Transactions on, vol. 15, no. 12, (2006), pp. 3736-3745.

[7] K. Dabov, A. Foi and V. Katkovnik, "Image denoising by sparse 3-D transform-domain collaborative filtering [J]”, Image Processing, IEEE Transactions on, vol. 16, no. 8, (2007), pp. 2080-2095.

[8] T. Huang, G. Yang and G. Tang, "A fast two-dimensional median filtering algorithm [J]", Acoustics, Speech and Signal Processing, IEEE Transactions on, vol. 27, no. 1, (2005), pp. 13-18. 
[9] A C. Bovik, T S. Huang and D C. Munson Jr., "A generalization of median filtering using linear combinations of order statistics [J]", Acoustics, Speech and Signal Processing, IEEE Transactions on, vol. 31, no. 6, (2006), pp. 1342-1350.

[10] Y H. Lee and S A. Kassam, "Generalized median filtering and related nonlinear filtering techniques [J]", Acoustics, Speech and Signal Processing, IEEE Transactions on, vol. 33, no. 3, (2006), pp. 672-683.

[11] M. Antonini, M. Barlaud and P. Mathieu, "Image coding using wavelet transform [J]", Image Processing, IEEE Transactions on, vol. 1, no. 2, (2007), pp. 205-220.

[12] A. Grinsted, J C. Moore and S. Jevrejeva, "Application of the cross wavelet transform and wavelet coherence to geophysical time series [J]", Nonlinear processes in geophysics, vol. 11, no. 5/6, (2004), pp. 561-566.

[13] H. Li, B S. Manjunath and S K. Mitra, "Multisensor image fusion using the wavelet transform [J]", Graphical models and image processing, vol. 57, no. 3, (2005), pp. 235-245.

[14] A S. Lewis and G. Knowles, "Image compression using the 2-D wavelet transform [J]", Image Processing, IEEE Transactions on, vol. 1, no. 2, (2006), pp. 244-250.

\section{Author}

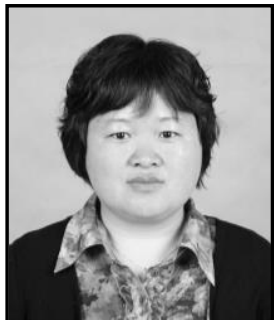

Jing Zhang, she received her Bachelor degree from Liaocheng University in Liaocheng, China and received her Master's degree from Shandong Normal University in Jinan, China. Now working in Dongchang College of Liao Cheng University, Associate Professor, Research Direction: Computer Application Technology. 